\title{
$666.95=60$ \\ sobre la naturaleza zeolítica de la toba amarilla de Gran Canaria
}

Habiendo realizado una serie de investigaciones que me han permitido poner de relieve la naturaleza zeolítica de algunas tobas volcánicas (1) pertenecientes a diferentes regiones eruptivas, y, en consecuencia, proporcionando algunos resultados acerca de su origen, trataré de dar cuenta en este trabajo de los resultados obtenidos sobre una muestra de toba amarilla de Gran Canaria (2), a fin de profundizar en los conocimientos sobre su constitución química y mineralógica.

La muestra de toba, litoide, objeto de esta investigación, ha sido enviada cortésmente por la Sociedad Cementos Especiales, S. A., a la que estoy agradecido. No dispongo de datos en relación con la estratigrafía de la zona de donde procede la muestra, ni una indicación precisa de su punto de toma. Sólo puedo añadir que dicha Sociedad la, emplea, después de una preparación adecuada, en la fabricación del cemento puzolánico.

La toba en estudio se ha sometido a las siguientes investigaciones: análisis químico, examen óptico y roentgenográfico; análisis térmico, ponderal y diferencial; ensayo de capacidad de cambio básico y prueba de "puzolanicidad". A continuación, referiremos ordenadamente los resultados.

\section{Análisis químico}

El análisis químico del material pulverizado ha dado el resultado referido en la tabla 1 , el cual, de acuerdo con el análisis óptico discutido más adelante, permite clasificar la roca en estudio como: traquita alcalina.

T A B L A 1

\begin{tabular}{cc} 
& $\begin{array}{c}\text { Toba de Gran } \\
\text { Canaria }\end{array}$ \\
$\mathrm{SiO}_{2}$ & 57,33 \\
$\mathrm{Al}_{2} \mathrm{O}_{3}\left(+\mathrm{TiO}_{2}+\mathrm{Mn}_{3} \mathrm{O}_{4}\right)$ & 18,08 \\
$\mathrm{FeO}_{2}$ & 0,15 \\
$\mathrm{Fe}_{2} \mathrm{O}_{3}$ & 4,40 \\
$\mathrm{CaO}$ & 1,44 \\
$\mathrm{MgO}$ & 0,76 \\
$\mathrm{~K}_{2} \mathrm{O}$ & 5,13 \\
$\mathrm{Na}_{2} \mathrm{O}$ & 6,30 \\
$\mathrm{H}_{2} \mathrm{O}^{+}$ & 6,28 \\
\hline & 99,87 \\
& 41,56 \\
Residuo al ataque ácido-básico suave (1). & \\
Residuo al ataque ácido-básico (Norma ita- \\
liana para la recepción de conglomeran- \\
tes hidráulicos).
\end{tabular}

(1) Se ha empleado clorhídrico $(1,5 \%)$, sucesivamente, solución de potasa al $5 \%$. El material de partida se ha refinado hasta pasar todo el por el tamiz de 10,0000 mallas $/ \mathrm{cm}^{2}$. Para las otras variables de la operación, es decir, cantidad de material, volumen de reactivos, temperatura del ensayo y duración, sirven las de la Norma Oficial ya mencionada (3). 

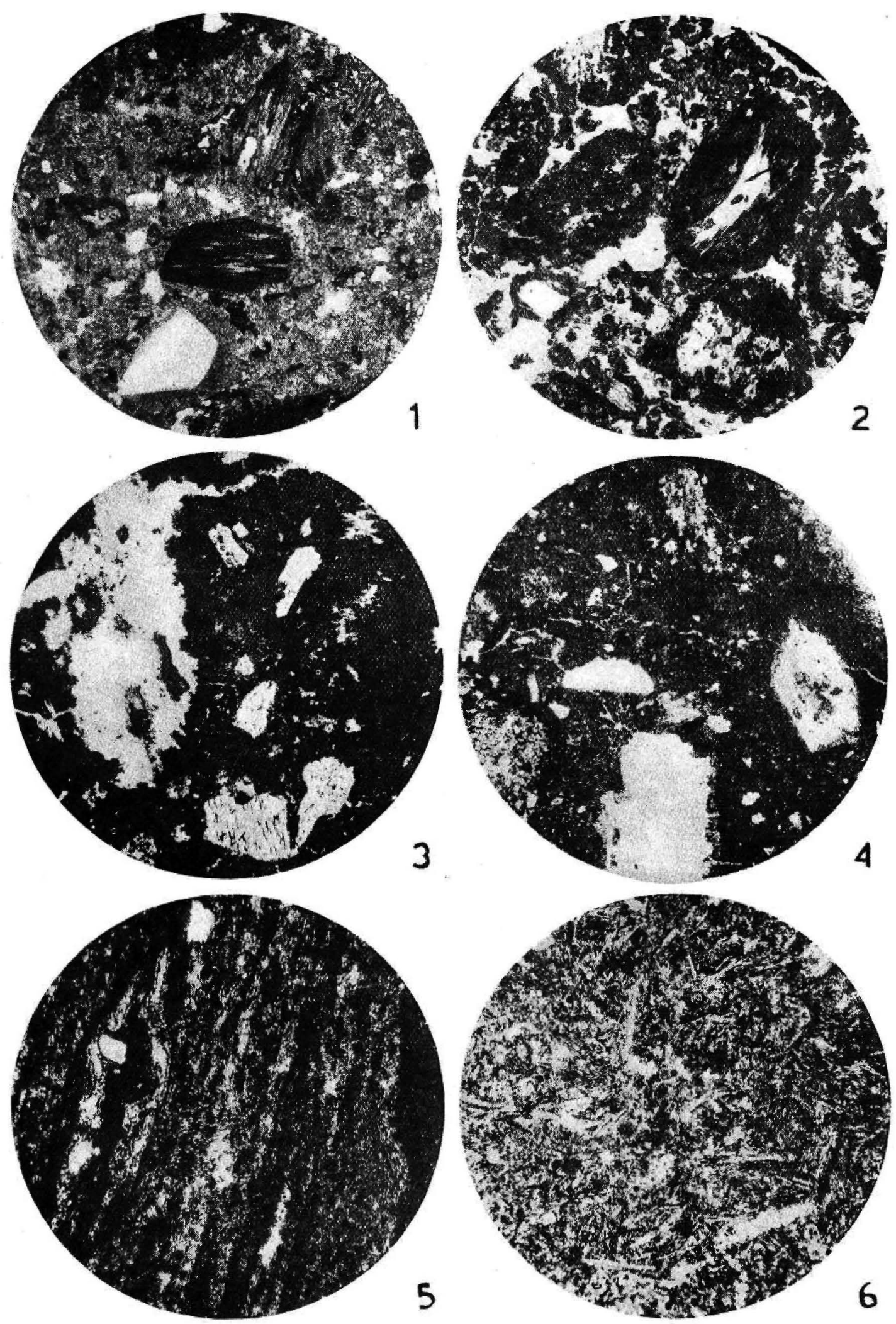

Láminł I.-Sección delgada; luz natural; aumento, X26; reducción a 19.

1. Toba amarilla napolitana, Variedad compacta. La sustancia intersticial, opaca, es poco diferente de los constituyentes principales, -2 . Toba amarilla napolitana. Variedad menos compacta. La sustancia intersticial es limpia.-3 y 4. Estructura de la toba amarilla de Gran Canaria. Agregado fundamental con fenocristales de santdino y pómez.-5. Toba de Gran Canaria. Intersticios del pómez rellenos de material secundario, entre ellos pequeños cristales birrefringentes (pertenecientes, con
de sanidino en la toba amarilla de Gran Canaria. 


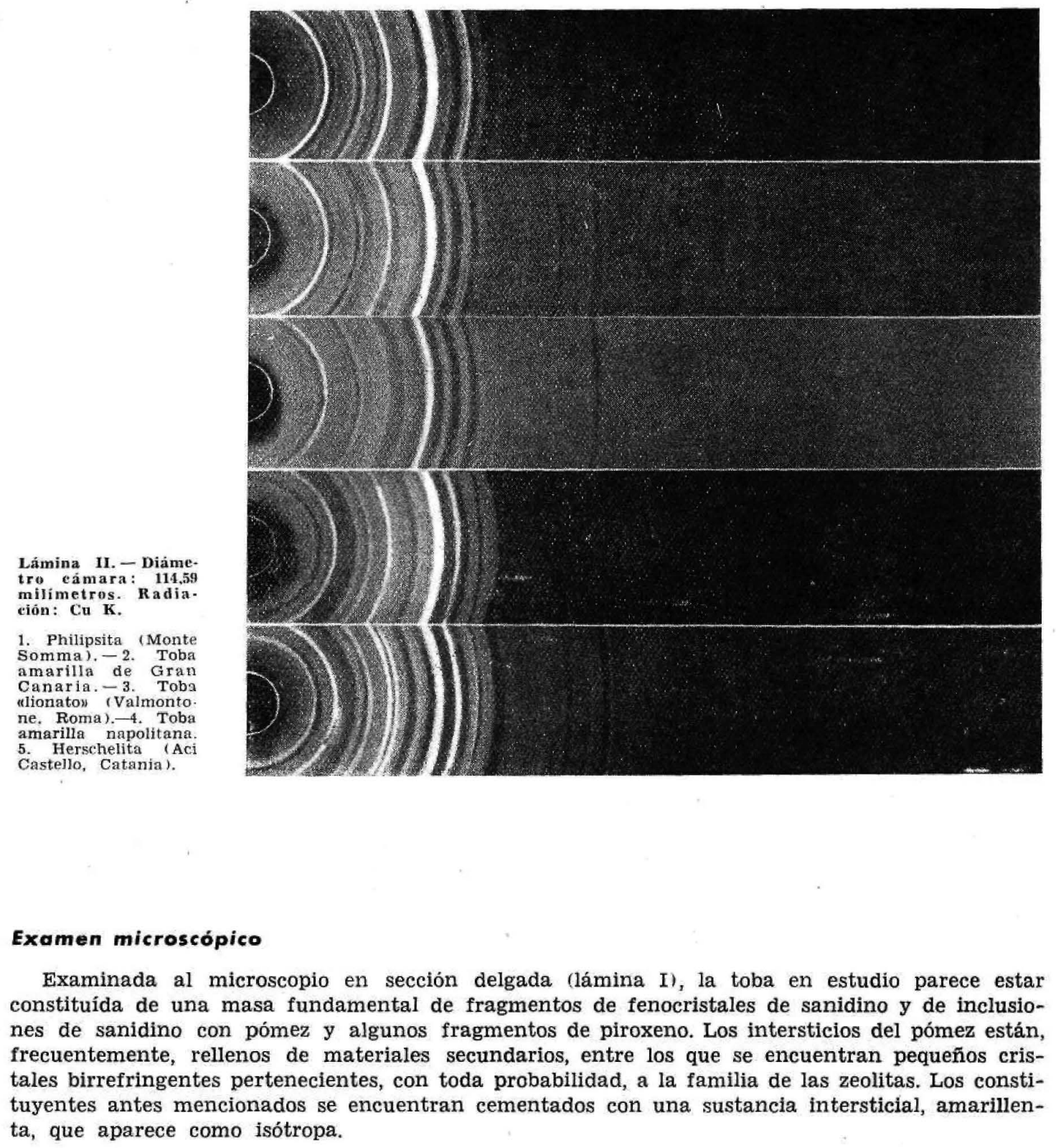

\section{Examen roentgenográfico}

El examen roentgenográfico (lámina II) se ha hecho sobre polvo sacado de la masa de fondo, cementante, de la toba en estudio, evitando en lo posible las inclusiones.

A diferencia de la investigación óptica, la roentgenográfica ha permitido realizar con toda evidencia que la masa de fondo, que aparece isótropa en el examen óptico en sección delgada, es de naturaleza zeolítica y que el término zeolítico predominante es la philipsita (4), análogamente a cuanto se ha puesto de manifiesto, siempre por vía roentgenográfica, para algunas muestras de toba "lionato" (5).

Como muestra la tabla 2, el espectograma de la toba de Gran Canaria es, en efecto, muy similar al obtenido con una muestra de philipsita del Monte Somma. En la tabla 2, se reúnen los datos que han servido para identificar el diagrama de difracción en estudio. 

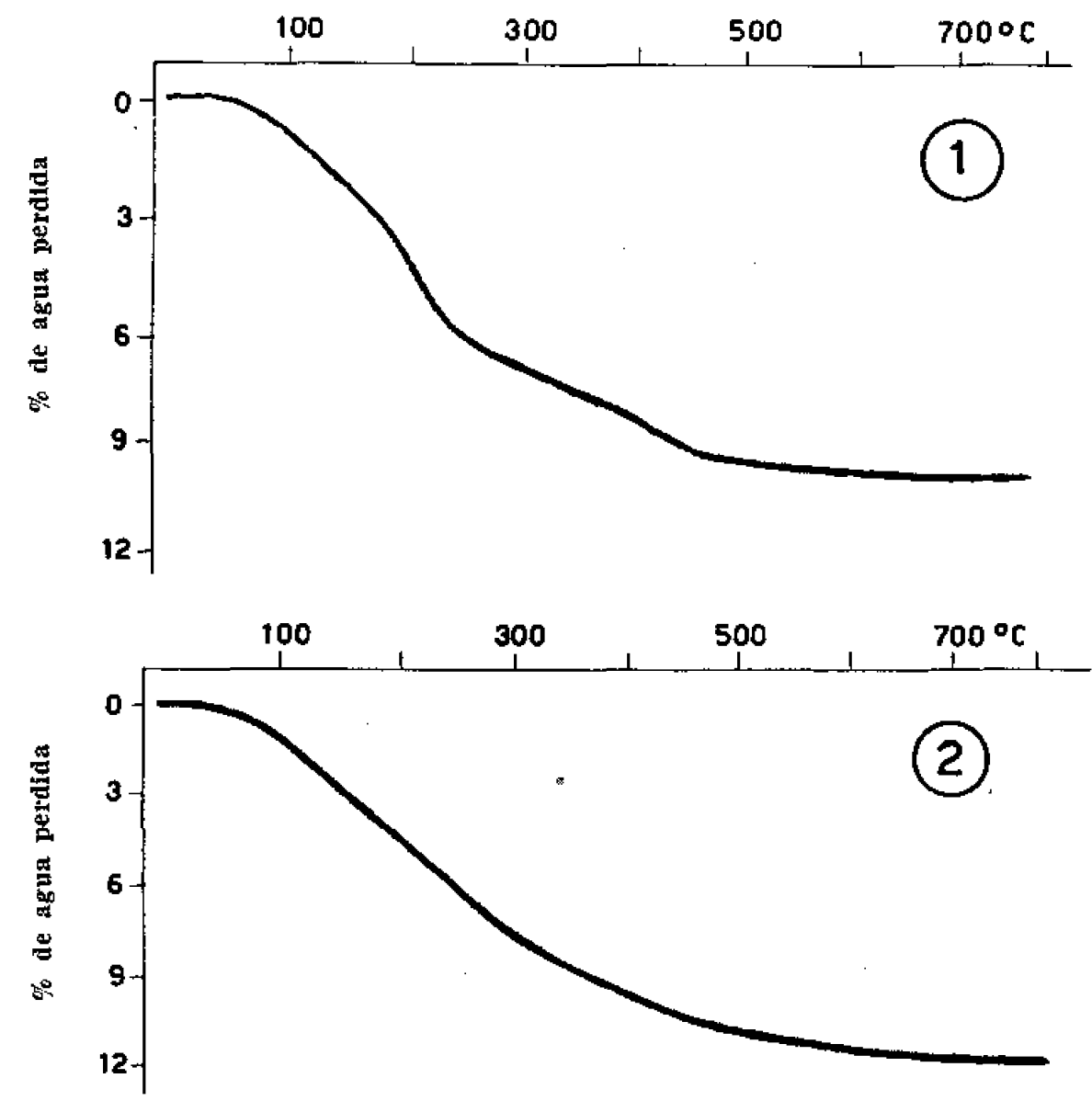

Fig. 1. - Diagramas pérde pesoltemperatura.

1. Toba amarilla de amarilla napolitana.

TA B L A 2

\begin{tabular}{cccc}
$\begin{array}{c}\text { Philipsita (Monie Somma) } \\
\text { Intensidad }\end{array}$ & \multicolumn{2}{c}{ Toba de Gran Canaria } \\
d en $\AA$ & Intensidad & d en $\mathbf{A}$ \\
md & 8,16 & md & 8,20 \\
f & 7,23 & f & 7,24 \\
m & 5,41 & m & 5,42 \\
mf & 4,98 & m & 5,01 \\
md & 4,32 & d & 4,34 \\
f & 4,14 & f & 4,15 \\
ff & 3,20 & ff & 3,20 \\
m & 2,93 & m & 2,94 \\
md & 2,75 & d & 2,75 \\
mf & 2,70 & d & 2,70 \\
mf & 1,96 & d & 1,97 \\
dl & 1,77 & d & 1,78 \\
d & 1,72 & d & 1,72
\end{tabular}

if $=$ muy fuerte; f - fuerte; $m f-$ semj-fuerte; n medla; mé.: media débil; d -..débil: dl=débll larga

\section{Anćlisis térmico-ponderal}

El anảlisis térmicco-ponderal ha permitido deducir el diagrama de la figura 1 , en su reproducción fotográfica original. En él se demuestra que la toba de Gran Canaria pierde el agua fljada flrmemente de modo muy uniforme, de acuerdo con su naturaleza zeolítica.

\section{Análisis térmico-diferencial}

El diagrama del análisis térmico-diferencial, representado en la figura 2, fija con mayor precisión la temperatura de deshidratación, que está precisamente comprendida en el Intervalo indicado en la curva termopondera!. 


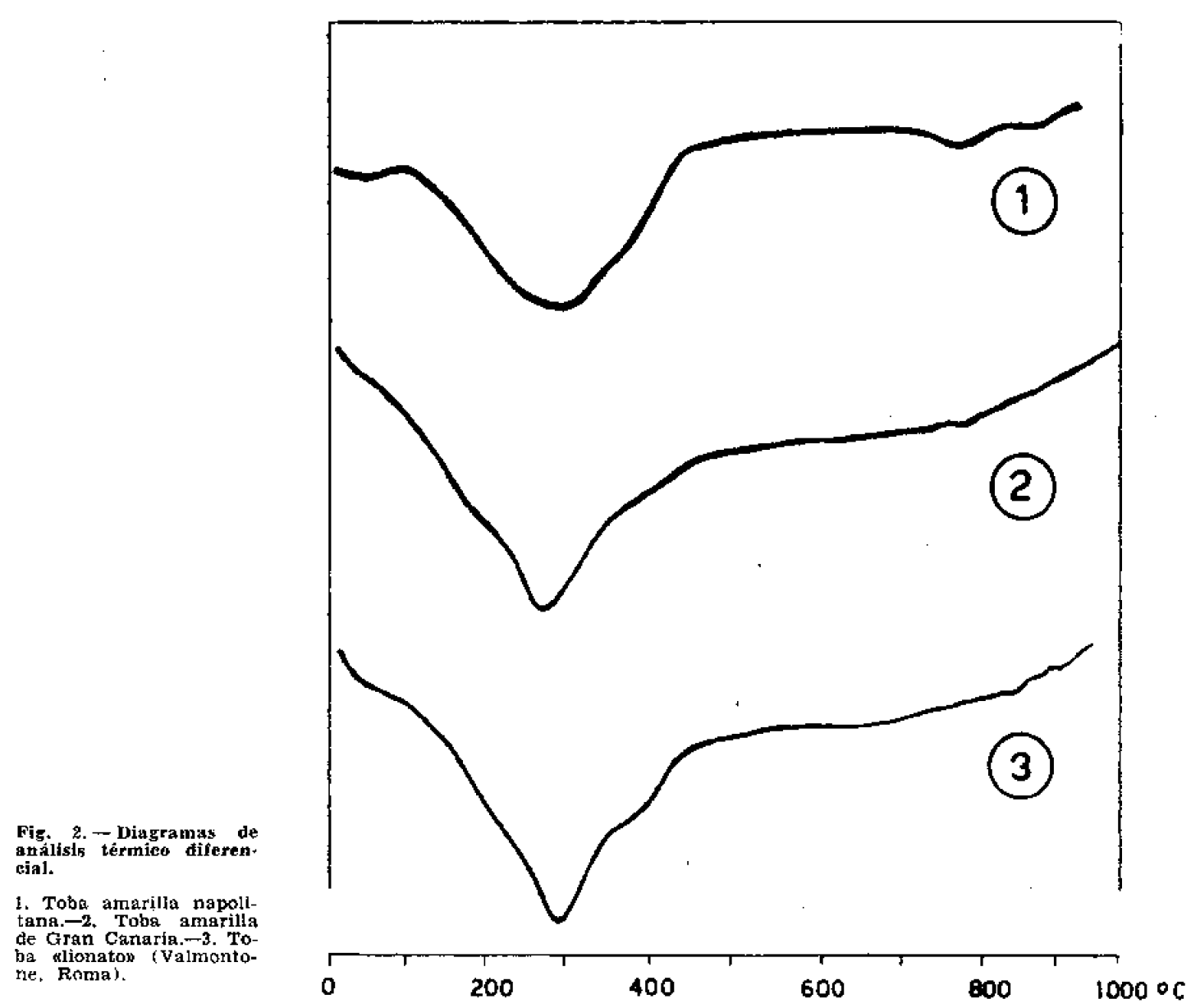

El diagrama se caracteriza por un notable efecto endotérmico, que comienza un poco por encima de la temperatura ambiente, termina alrededor de $400^{\circ} \mathrm{C}$ y presenta un pico hacia los $300^{\circ} \mathrm{C}$. Esto debe atribuirse a la expulsión del agua de la red del mineral zeolítieo (philipsita). Aparte de otros detalles, la marcha general del diagrama térmico-diferencial es análogo al que se registra, en ldénticas condiciones, para otras tobas volcánicas, entre las cuales se encuentran: una muestra de toba, llamada "lionato", de la región volcánica de las Colinas de Albania (6), en la que el término zeolitico predominante es también la philipsita, y una muestra de toba amarilla napolitana, en la que el constituyente zeolitico es, en su lugar, la herschelita. Los diagramas de estas dos últimas tobas se refieren en la figura 2 para facilltar la comparación.

\section{Valoración de le capacidad de cambio de base}

La determinación se ha seguido mezclando la muestra de la toba de Gran Canaria, prevlamente pulverizada hasta pasar toda ella por el tamiz de 10.000 mallas por centimetro cuadrado, con solución de cloruro amónjco caproximadamente $\mathrm{N} / 10$ ) y observando la misma técnica referida en un trabajo anterior (7).

En la tabla 3 se resumen los datos analíticos de las pruebas realizadas, con las cuales se ha deducido el gráfico de la flgura 3 . 


\section{MILIEQUIVALENTES}

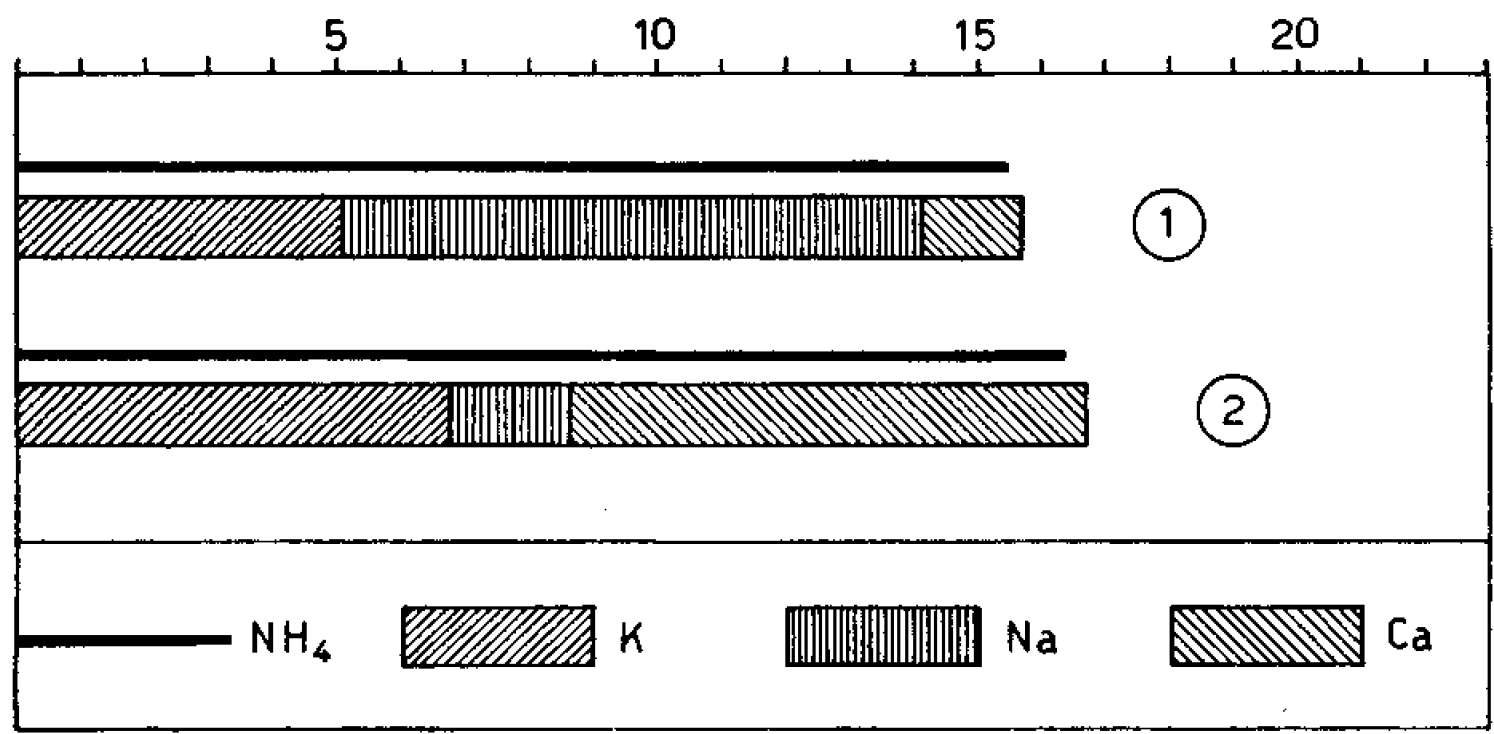

Fig. 3.-Fepresentación de los resultados de la prueba de cambin de base, expresados en la tabla 3 .

Como muestra la tabla 3, la toba de Gran Canaria denuncia una sensible actividad de cambio, de acuerdo con su carácter zeolítico.

TA BLA $\mathbf{3}$

Capacldad de cambio básico en solución de $\mathrm{NH}, \mathrm{Cl}$ (aproximadamente $\mathrm{N} / 1 \mathrm{1}$ )

\begin{tabular}{|c|c|c|c|c|c|c|}
\hline \multirow{2}{*}{\multicolumn{2}{|c|}{ MUESTRA DE TOBA }} & \multirow{2}{*}{$\begin{array}{c}\text { Miliequivalentes } \\
\text { de NH, } \\
\text { separados de la } \\
\text { solueion }\end{array}$} & \multicolumn{3}{|c|}{$\begin{array}{l}\text { Miliequivalentes gue han } \\
\text { pasado a la solución }\end{array}$} & \multirow{2}{*}{$\begin{array}{l}\text { Total de oquiva } \\
\text { lestes de camblo }\end{array}$} \\
\hline & & & $\mathbf{K}$ & $\mathrm{Na}$ & $\mathrm{Ca}$ & \\
\hline & Toba de Gran Canaria. & 15,50 & 5,11 & 9,13 & 1,50 & 15,74 \\
\hline & Toba amarilla napolitana. & 16,47 & 6,81 & 1,71 & 8,15 & 16,67 \\
\hline
\end{tabular}

\section{Ensayo de "puzolanicidad"}

Asegurado por medio del análisis roentgenogräfico, y confirmado con las investigaciones complementarias que la toba de Gran Canaria es de naturaleza zeolitica, he creido útil someterla tambièn al erisayo de "puzolanicidad" (8) para valorar su actividad en la fljación de la cal. Se ha seguido la misma técnica con la que se han ensayado otros materiales con comportamiento "puzolánico" (9), es decir, preparando un cemento "puzolánico" por mezcla de la toba en estudio (pasada por el tamiz de 10.000 mallas $/ \mathrm{cm}^{2}$ ) con clínker de Portland de finura similar. Las proporciones empleadas en la preparación de la mezcla son: $65 \%$ de clínker y $35 \%$ de toba de Gran Canaria.

Se ha seguldo la técniça que el ensayo anteriormente mencionado prescribe, y en consecuencia, $20 \mathrm{~g}$ de este cemento, adicionados de $100 \mathrm{~cm}^{3}$ de agua destilada, se colocaron en un vaso de vidrio parafinado, y después de bien tapado se mantiene a $40^{\circ} \mathrm{C}$ durante ocho días. Pasado este tiempo se determina en el líquido limpio, eliminada la pasta de cemento, la alcalinidad total y el título de hidróxido cáléco. 
Alealinidad total de la solución de contacto con la pasta de cemento en estudio (milïmoles $0 \mathrm{H} / \mathrm{litro}$ )

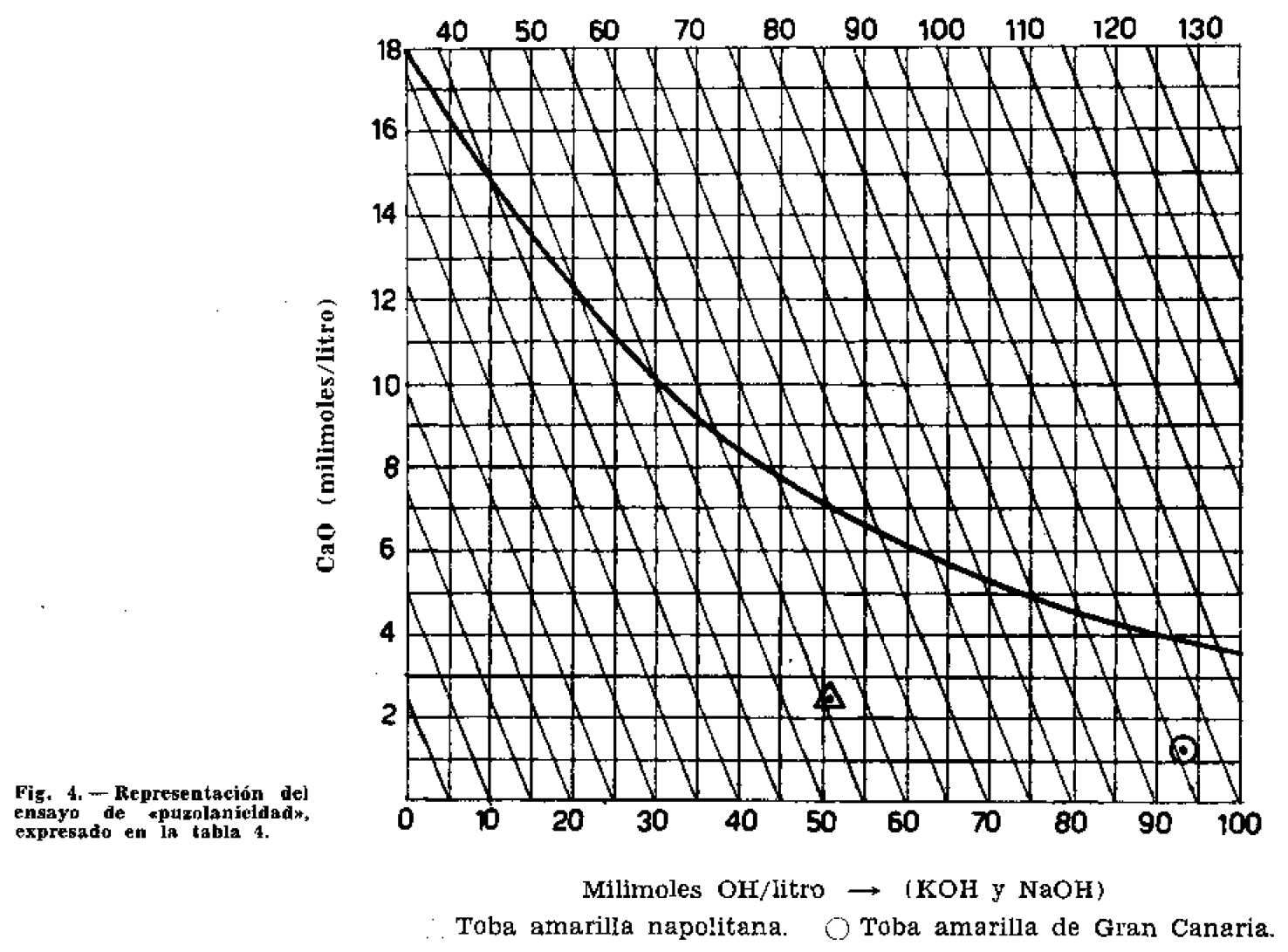

Si el material presenta actividad "puzolánica"-como en este caso-, y si la relación de mezcla está convenientemente ajustada, el punto representativo de Ia concentración en cal de la solución debe montarse debajo de la isoterma de solubilidad de la propia cal en presencia de álcalis. En la tabla 4 se encuentran los datos analíticos que ha proporcionado el ensayo, que corresponden con los de la flgura 4. El término de comparación es una toba volcánica de comportamiento "puzolánico": la toba amarilla napolitana. Los resultados demuestran que el cemento "puzolánico" preparado empleando la toba de Gran Canaria, de modo similar al obtenido con la toba amarilla napolitana, no es capaz-en idénticas condiciones, con la técnlẹ y en el plazo que el ensayo establece-de saturar de hidróxido cálcico la solución de contacto.

Por lo tanto, la toba amarilla de Gran Canaria es también, desde el punto de vista técnico, una buena "puzolana".

T A B L A 4

$\begin{array}{lcc} & \begin{array}{c}\text { ol } \\ \text { (milimoles/litra) }\end{array} & \begin{array}{c}\text { Cao } \\ \text { (milimales/litro) }\end{array} \\ \begin{array}{l}\text { Clinker/toba amarilla de Gran Ca- } \\ \text { naria. }\end{array} & \$ 5,31 & 1,28 \\ \text { Clinker/toba amarilla napolitana. } & 55,15 & 2,42\end{array}$

(Mezcla de $65 \%$ de clinker de Portland y $35 \%$ de material con actividad "puzolánica", ambos pasados por el tamiz de 10.000 mallas $/ \mathrm{cm}^{2}$ ) 
El conjunto de resultados experimentales coincide en señalar que la masa de fondo conglomerante de la toba amarilla de Gran Canaria, tiene por constituyente principal la philipsita. Este resuitado demuestra que también para la toba en estudio existe un proceso de zeolitización en la base de su origen, de modo análogo a cuanto se ha puesto de manifiesto por otras tobas volcánlcas $(10)$.

El término zeolitico, precominante en la masa de fondo, ha tenido su origen en la base vitrea, altamente reactiva, de los materiales piroclásticos sometidos a acciones hidrotermales o neumatolíticas.

En la toba amarilla de Gran Canaria son, por to tanto, comunes las caracteristicas peculiares de las "tobas zeolíticas"; en primer término, la actividad de fijación de la cal (puzolanicidady:

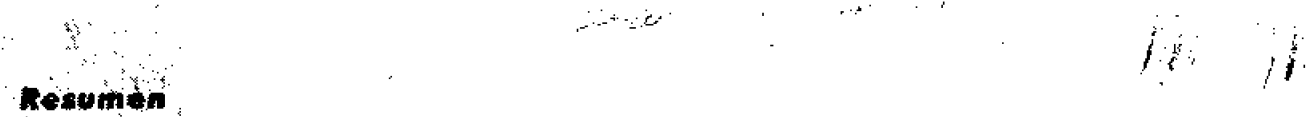

Se demuestra que el constituyente princlpal de la masa de fondo cementante de la toba en estudio, es un mineral zeolitico: la philipsita, y que también en la toba amarilla de Gran Canaria son comunes las caracteristicas pecullares de las "tobas zeolíticas", entre las cuales se encuentra en primer lugar la "puzolanteidad".

\section{Refractarios para flormos de Cemento}

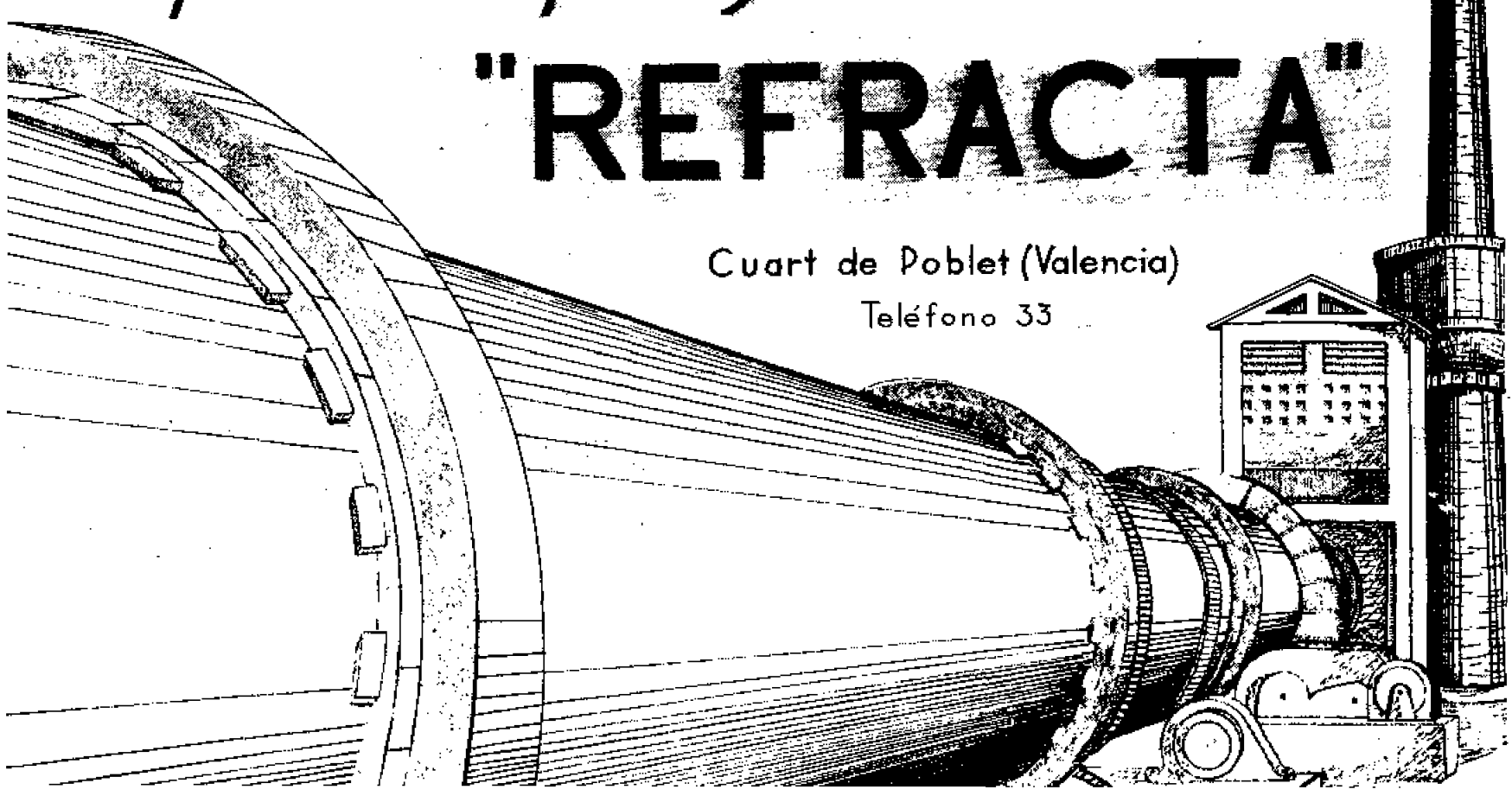

